\title{
Original
}

\section{Membrane Effects of Monoamine Oxidase Inhibitors on Erythrocytes and Freshly Isolated Hepatocytes}

\author{
Mayumi TsuJi, Kyoko Kodama, Daichi MorioKa, \\ Masako OKAZAKI and Katsuji OgUCHI
}

\begin{abstract}
Effects of monoamine oxidase (MAO) inhibitors on two kinds of membranes, biological and model membranes, were studied. Erythrocytes and freshly isolated hepatocytes from rats were used to examine the effects of MAO inhibitors on biological membranes. Hydrazide and hydrazine derivatives, except isonicotinic acid, enhanced hemolysis and the stability of isolated hepatocytes membrane. Deprenyl and moclobemide stabilized the biological membranes of erythrocytes and isolated hepatocytes, and moclobemide induced slight enzyme leakage from isolated hepatocytes. Clorgyline and brofaromine had opposite effects. At lower concentrations, they remarkably inhibited hemolysis and enzyme leakage. At higher concentrations, they enhanced hemolysis and apparent enzyme leakage. To investigate effects of MAO inhibitors on a model membrane, changes of phase transition temperature of dipalmitoyl phosphatidylcholine liposomes was determined. Brofaromine induced the most extreme decline of phase transition temperature. Deprenyl and clorgyline also induced moderate decline of transition temperature, and phenylhydrazine and $p$-tolylhydrazine induced mild decline. These results suggest that the effects of clorgyline and brofaromine on the membranes of erythrocytes and freshly isolated hepatocytes might be partly due to increased fluidity of membrane phospholipid. These severe membrane effects of MAO inhibitors might be the reason for their cytotoxicity.
\end{abstract}

Key words: monoamine oxidase inhibitor, membrane, hepatocyte, erythrocyte, liposome, fluidity

\section{Introduction}

There have been many reports of the adverse effects of antidepressant drugs such as monoamine oxidase (MAO) inhibitors ${ }^{1-5)}$ and tricyclic antidepressants ${ }^{4-6}$. The discovery of MAO-A and MAO-B types of the MAO enzyme, each showing distinctly different substrate preferences and inhibitor sensitivity ${ }^{i}$, has led to an increase in the development of new and selectively acting MAO-A or MAO-B inhibitors without the hydrazine structure. The cytotoxicity of various therapeutic compounds that induce membrane action has already been reported. We previously demonstrated that the cytotoxicity of some compounds, such as tricyclic antidepressants, is positively correlated between hepatocytes and erythrocytes ${ }^{8)}$. We also reported that the membrane stabilizing action on rat erythrocytes by some compounds was related to increased fluidity of the phospholipid bilayer ${ }^{9)}$. Therefore, the effects of 
therapeutic drugs on cell membrane action are clinically interesting.

The present study aims to compare the effects of MAO inhibitors on the hypotonic hemolysis of erythrocytes and leakage of enzymes from freshly isolated hepatocytes. In addition, the effects of MAO inhibitors on a model membrane were investigated to measure changes of phase transition temperature using dipalmitoyl phosphatidylcholine (DPPC) liposomes.

\section{Materials and Methods}

\section{Materials and animals}

Iproniazid was obtained from Tokyo Kasei Kogyo Co., Ltd. (Tokyo, Japan); phenylhydrazine was obtained from Wako Pure Chemical Industries Ltd. (Osaka, Japan); deprenyl was provided by Dr. J. Konoll of Semmelweis University, School of Medicine, (Budapest, Hungary); isonicotinic acid and pargyline were obtained from Sigma Chemical Co., Ltd. (St. Louis, U.S.A.); clorgyline was provided by May \& Baker Ltd. (Dagenham, U.K.); brofaromine (CGP11305A) was provided by Ciba-Geigy Ltd. (Basel, Switzerland); moclobemide was provided by Hoffmam-La Roche \& Co., Ltd. (Basel, Switzerland).

Collagenase (type IV) and DPPC were obtained from Sigma Chemical Co., Ltd. (St. Louis, U.S.A.). William's E medium was purchased from Flow Laboratories (Irvine, Scotland). Trypan blue $(0.4 \%)$ was obtained from Gibco Laboratories Life Technologies Inc. (New York, U.S.A.). All other chemicals used in this experiment were the purest grade commercially available. In the present study, male Sprague-Dawley rats (6 weeks; $150 \mathrm{~g}$ ) were used.

\section{Studies of hypotonic hemolysis}

Effects of MAO inhibitors on hypotonic hemolysis of rat erythrocytes were studied as previously described ${ }^{10)}$. MAO inhibitors were dissolved in a hypotonic buffer (about $54 \mathrm{mM}$ $\mathrm{NaCl}$ in $10 \mathrm{mM}$ phosphate buffer, $\mathrm{pH} 7.4$ ) which usually induced lysis of $50 \%$ of the erythrocytes. The erythrocyte suspension $\left(2 \times 10^{9}\right.$ cells $\left./ \mathrm{ml}\right)$ was incubated for $1 \mathrm{hr}$ at $37^{\circ} \mathrm{C}$ with various concentrations of MAO inhibitors ranging from $1 \times 10^{-5}$ to $1 \times 10^{-3} \mathrm{M}$.

\section{Isolated hepatocytes of rat}

Hepatocytes were isolated from rats by a collagenase perfusion technique ${ }^{11)}$, and viable hepatocytes were suspended in a mixture of William's E medium, supplemented as previously described $^{10)}$. The viability of isolated hepatocytes determined by trypan blue exclusion was in the range of $90-95 \%$. MAO inhibitors in a range of $1 \times 10^{-6} \mathrm{M}$ to $1 \times 10^{-3} \mathrm{M}$ were dissolved in the William's E medium. The isolated hepatocyte suspension $\left(1 \times 10^{5} \mathrm{cells} / \mathrm{ml}\right)$ was incubated for $1 \mathrm{hr}$ at $37^{\circ} \mathrm{C}$ with MAO inhibitors. Activities of glutamic oxaloacetic transaminase (GOT) and glutamic pyruvic transaminase (GPT) which leaked from the isolated hepatocytes were determined by the method of Ohkawa et al. ${ }^{12)}$. The morphological changes of isolated hepatocytes treated with MAO inhibitors were observed under a phasecontrast microscope after dying with trypan blue.

Phase-transition temperature study

DPPC at $1 \mathrm{mM}$ concentration was dispersed in $10 \mathrm{mM}$ phosphate buffer $(\mathrm{pH}$ 7.4) using a Sonifier cell disruptor 200 (Branson), as previously described ${ }^{9)}$. This method has been generally accepted to produce liposomes of DPPC. MAO inhibitors ranging from $1 \times 10^{-6}$ to $1 \times 10^{-3} \mathrm{M}$ were dissolved in the DPPC liposomal suspension. The phase transition temperature of each liposomal suspension with MAO inhibitors was measured to an accuracy of $0.01^{\circ} \mathrm{C}$ with a thermistor probe (Takara Thermistor Instruments, Co., Ltd.). The midpoint 
between the beginning of the decrease in the scattering and reaching a plateau was taken as the phase transition temperature for each concentration of MAO inhibitors. A decrease in phase transition temperature without MAO inhibitors was plotted against the concentration of each MAO inhibitor. The concentration of each MAO inhibitor necessary to induce a $1.0^{\circ} \mathrm{C}$ decline of phase transition temperature was calculated from the midpoint of each slope.

Statistical significance of the difference between the control and test values was calculated by Student's $t$-test.

\section{Results}

Effects of MAO inhibitors on the hypotonic hemolysis of rat erythrocytes are shown in Fig. 1. In the present study, the value of about $50 \%$ hypotonic hemolysis without treatment of MAO inhibitors was used as the control. Treatment with iproniazid at a concentration range of $2 \times 10^{-4}$ to $1 \times 10^{-3} \mathrm{M}$ enhanced hemolysis significantly more than the control. In the treatment with $p$-tolylhydrazine, slight protection of hemolysis was observed at a con-

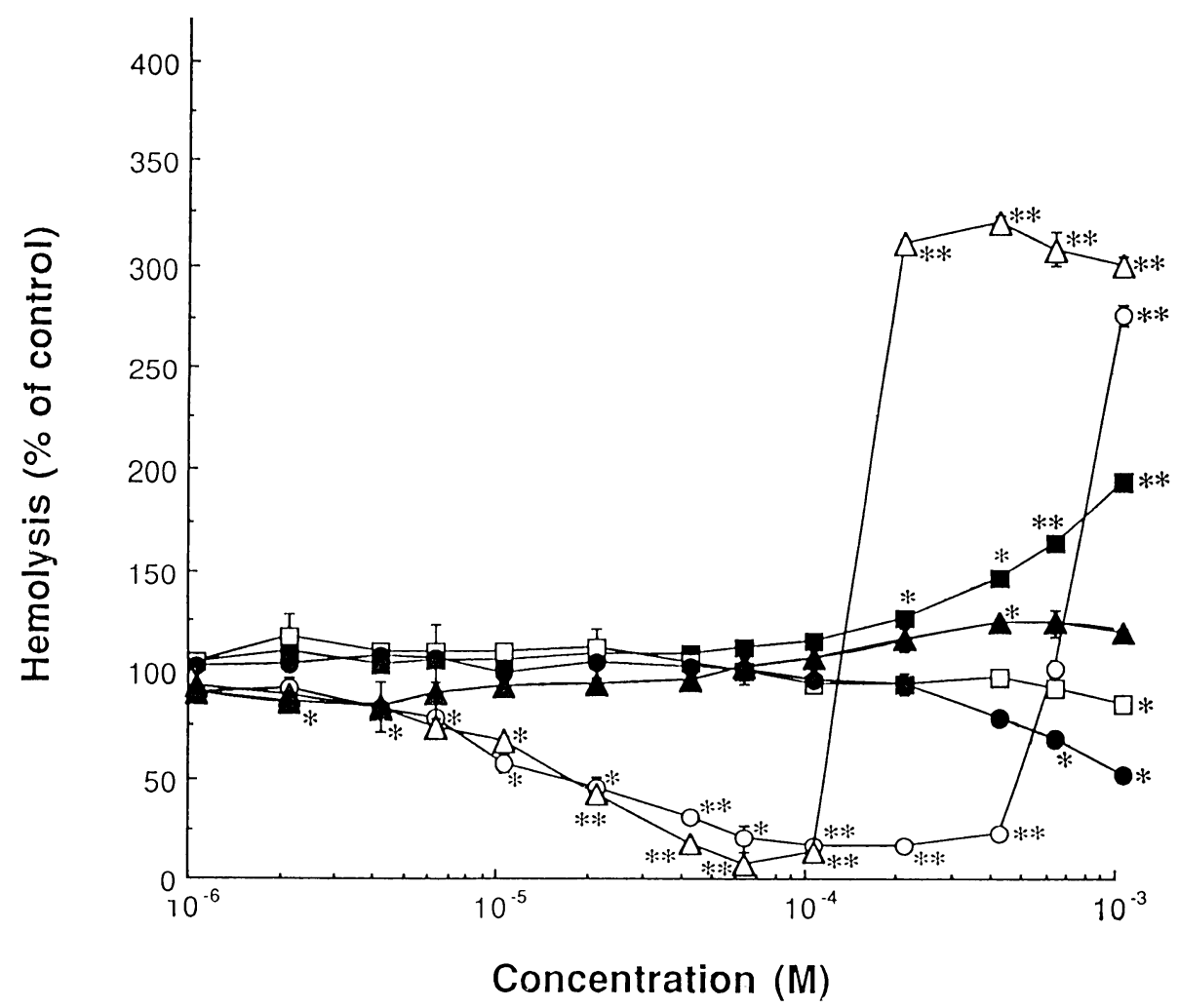

Fig. 1. Effect of MAO inhibitors on hypotonic hemolysis of rat erythrocytes. Erythrocyte suspension was incubated for $1 \mathrm{hr}$ at $37^{\circ} \mathrm{C}$ with various concentrations of MAO inhibitors in a range of $1 \times 10^{-6} \mathrm{M}$ to $1 \times 10^{-3} \mathrm{M}$. Data are expressed as percentage of the mean value of control. Statistically significant changes are indicated by: $* p<0.01$ and $* * p p<0.001$ compared to the values obtained from the control (Student's $t$-test).

$\boldsymbol{\square}-\mathbf{\square}$, iproniazid; $\mathbf{\Delta}-\mathbf{\Delta}$, p-tolylhydrazine; $\bullet-\bullet$, deprenyl; $\Delta-\Delta$, clorgyline; $\bigcirc-\bigcirc$, brofaromine; $\square-\square$, moclobemide. 
Table 1. Effect of monoamine oxidase inhibitors on leakage of glutamic oxaloacetic transaminase from freshly isolated hepatocytes.

\begin{tabular}{|c|c|c|c|c|}
\hline \multirow{2}{*}{ Compounds } & \multicolumn{4}{|c|}{ Concentrations } \\
\hline & $10^{-6} \mathrm{M}$ & $10^{-5} \mathrm{M}$ & $10^{-4} \mathrm{M}$ & $10^{-3} \mathrm{M}$ \\
\hline & & $\%$ & trol & \\
\hline \multicolumn{5}{|l|}{ Hydrazines } \\
\hline Isonicotinic acid & $106.3 \pm 14.9$ & $104.8 \pm 17.3$ & $117.7 \pm 23.6^{*}$ & $126.5 \pm 34.6^{*}$ \\
\hline Iproniazid & $100.3 \pm 22.8$ & $109.8 \pm 13.0$ & $95.0 \pm 24.1$ & $85.3 \pm 7.8$ \\
\hline Phenylhydrazine & $95.7 \pm 10.7$ & $112.8 \pm 27.7$ & $100.1 \pm 18.6$ & $49.8 \pm 14.6^{* * *}$ \\
\hline$p$-Tolylhydrazine & $93.5 \pm 8.8$ & $94.7 \pm 19.0$ & $83.1 \pm 27.8$ & $30.7 \pm 4.8 * * *$ \\
\hline \multicolumn{5}{|l|}{ Propinylamines } \\
\hline Deprenyl & $95.0 \pm 13.3$ & $94.4 \pm 11.8$ & $74.2 \pm 10.4^{* * *}$ & $83.1 \pm 8.0 * * *$ \\
\hline Pargyline & $96.0 \pm 14.9$ & $104.8 \pm 22.8$ & $112.0 \pm 1.8$ & $101.2 \pm 39.8$ \\
\hline Clorgyline & $72.6 \pm 6.2 * *$ & $97.2 \pm 24.7$ & $500.8 \pm 61.8 * * *$ & $1337.8 \pm 70.4^{* * *}$ \\
\hline \multicolumn{5}{|l|}{ Others } \\
\hline Brofaromine & $94.4 \pm 27.7$ & $96.9 \pm 31.3$ & $179.6 \pm 67.6$ & $582.0 \pm 22.7 * * *$ \\
\hline Moclobemide & $98.3 \pm 19.6$ & $115.2 \pm 12.6$ & $103.8 \pm 9.5$ & $117.9 \pm 24.0 *$ \\
\hline
\end{tabular}

Hepatocytes were suspended in William's $\mathrm{E}$ medium and incubated for $1 \mathrm{hr}$ at $37^{\circ} \mathrm{C}$ after the addition of various concentrations in a range of $1 \times 10^{-6}$ to $1 \times 10^{-3} \mathrm{M}$. Data are expressed as percentage of the mean value of the control. Each value represents the mean \pm SD of 5 to 10 samples. Significant differences from the control value are: ${ }^{*} p<0.05,{ }^{*} p<0.01$ and ${ }^{* * *} p<0.001$ (Student's $t$-test).

Table 2. Effect of monoamine oxidase inhibitors on leakage of glutamic pyruvic transaminase from freshly isolated hepatoctyes.

\begin{tabular}{lcccc}
\hline & \multicolumn{4}{c}{ Concentrations } \\
\cline { 2 - 4 } Compounds & $10^{-6} \mathrm{M}$ & $10^{-5} \mathrm{M}$ & $10^{-4} \mathrm{M}$ & $10^{-3} \mathrm{M}$ \\
\hline & & $\%$ of control \\
Hydrazines & & & & \\
$\quad$ Isonicotinic acid & $114.5 \pm 17.5$ & $104.0 \pm 19.2$ & $107.6 \pm 32.9$ & $115.0 \pm 7.3^{* *}$ \\
Iproniazid & $107.0 \pm 18.7$ & $119.4 \pm 23.2$ & $101.0 \pm 20.8$ & $64.6 \pm 7.3^{*}$ \\
Phenylhydrazine & $120.4 \pm 3.6$ & $89.6 \pm 27.5$ & $75.7 \pm 32.7$ & $39.3 \pm 2.8^{* * *}$ \\
p-Tolylhydrazine & $94.5 \pm 13.9$ & $96.0 \pm 29.5$ & $53.6 \pm 7.6 * * *$ & $6.7 \pm 7.3 * * *$ \\
Propinylamines & & & & \\
Deprenyl & $109.6 \pm 19.2$ & $106.6 \pm 14.1$ & $88.8 \pm 14.9$ & $90.6 \pm 9.7$ \\
Pargyline & $99.3 \pm 10.8$ & $94.2 \pm 20.4$ & $80.4 \pm 4.2 * * *$ & $70.2 \pm 10.0^{* * *}$ \\
Clorgyline & $87.7 \pm 17.8$ & $88.3 \pm 12.9 *$ & $202.2 \pm 30.8 * *$ & $438.4 \pm 29.7 * * *$ \\
& & & & \\
Others & & & & \\
Brofaromine & $106.5 \pm 16.5$ & $86.7 \pm 10.7 *$ & $94.3 \pm 11.4$ & $370.3 \pm 18.4 * * *$ \\
Moclobemide & $100.8 \pm 12.8$ & $106.0 \pm 15.8$ & $94.7 \pm 13.3$ & $97.1 \pm 42.4$ \\
\hline
\end{tabular}

Conditions are shown in Table 1. Each value represents the mean \pm SD of 5 to 10 samples. Significant differences from the control value are: ${ }^{*} p<0.05,{ }^{* *} p<0.01$ and ${ }^{* * *} p<0.001$ (Student's $t$-test).

centration of $1 \times 10^{-6}$ to $6 \times 10^{-3} \mathrm{M}$, while slight enhancement of hemolysis was observed at more than $2 \times 10^{-4} \mathrm{M}$. However, treatment with deprenyl at $4 \times 10^{-4}$ to $1 \times 10^{-3} \mathrm{M}$, and with moclobemide at $1 \times 10^{-3} \mathrm{M}$ induced protection of hypotonic hemolysis. Clorgyline and brofaromine induced opposite effects on hypotonic hemolysis: 1) Treatment with $2 \times 10^{-6}$ to 


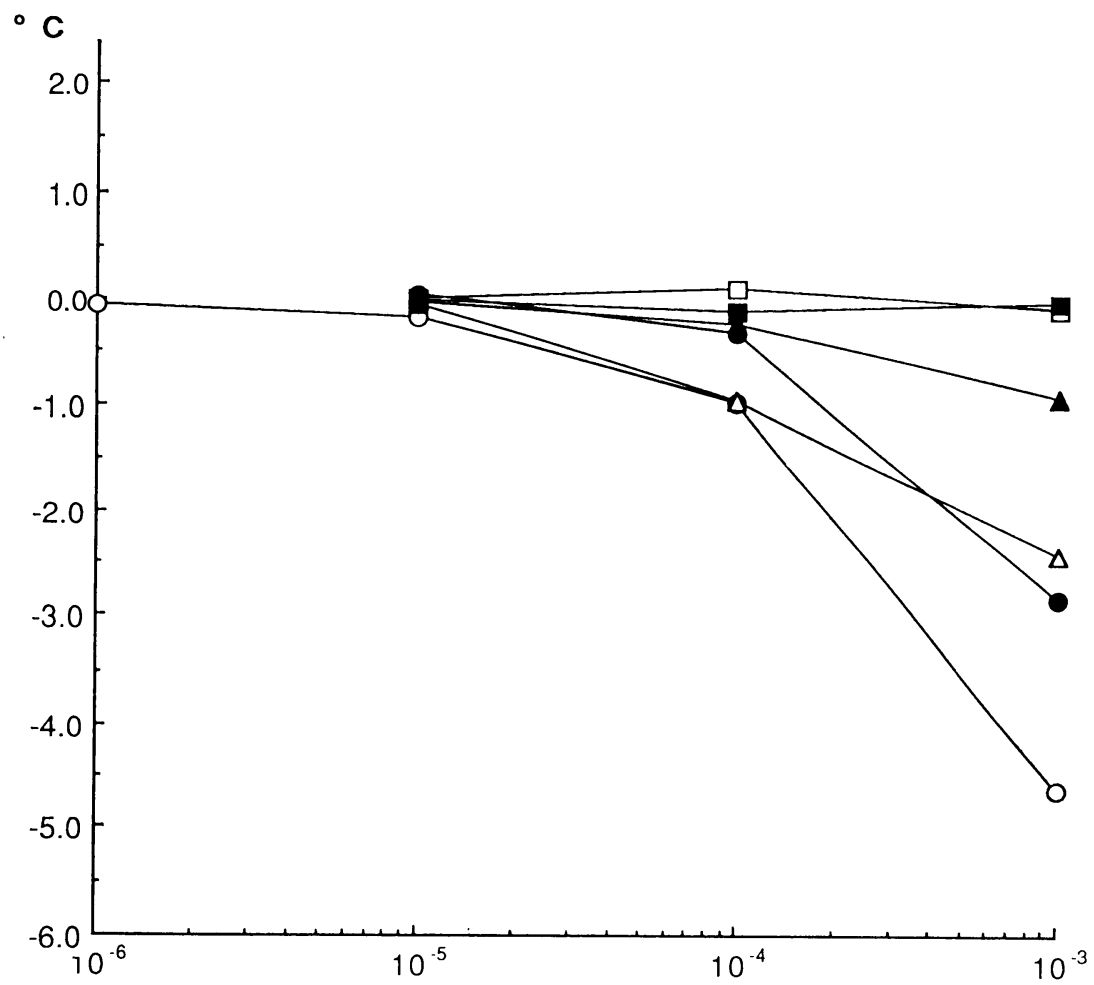

\section{Concentration (M)}

Fig. 2. Effect of MAO inhibitors on phase transition temperature of $1 \mathrm{mM}$ DPPC liposomal suspension. Data are expressed as the decline in temperature from the phase transition temperature of the control.

$\square-\mathbf{\square}$, iproniazid; $\Delta-\mathbf{\Lambda}, p$-tolylhydrazine; $\bullet-\bullet$ deprenyl; $\Delta-\Delta$, clorgyline; $\bigcirc$ ○, brofaromine; $\square-\square$, moclobemide.

$1 \times 10^{-4} \mathrm{M}$ clorgyline or with $4 \times 10^{-6}$ to $4 \times 10^{-4} \mathrm{M}$ brofaromine inhibited hypotonic hemolysis. 2) They substantially enhanced hypotonic hemolysis about $296 \%$ and $270 \%$, respectively at the higher concentrations.

Effects of MAO inhibitors on the leakage of GOT and GPT from rat isolated hepatocytes are shown in Tables 1 and 2. In this study, isolated hepatocytes untreated MAO inhibitors were used as control. The amounts of GOT and GPT leaked from control isolated hepatocytes were $22.8 \pm 7.2$ (mean $\pm \mathrm{SD}$ ) $\mathrm{KU} / \mathrm{ml}$ and $5.8 \pm 2.2 \mathrm{KU} / \mathrm{ml}$, respectively. In the isolated hepatocytes treated with iproniazid and pargyline, there was no apparent effect on the leakage of GOT. The treatment with isonicotinic acid at concentrations of $10^{-4}$ and $10^{-3} \mathrm{M}$, or with moclobemide at $10^{-3} \mathrm{M}$, induced a significantly greater increase in GOT leakage than that from the control. Treatment with $10^{-i} \mathrm{M}$ phenylhydrazine or $p$-tolylhydrazine decreased GOT leakage to less than that of control. In the isolated hepatocytes treated with $10^{-6} \mathrm{M}$ clorgyline, a significant decrease in GOT leakage was observed. At $10^{-4} \mathrm{M}$ and $10^{-3} \mathrm{M}$, clorgyline induced increases of about $500 \%$ and $1337 \%$, respectively. Treatment with brofaromine at $10^{-3} \mathrm{M}$ significantly increased GOT leakage from isolated hepatocytes. Treatment with deprenyl or 
Table 3. Effects of monoamine oxidase inhibitors on biological membranes and model membrane.

\begin{tabular}{|c|c|c|c|c|c|}
\hline \multirow{2}{*}{ Compounds } & \multicolumn{2}{|c|}{ Erythrocytes } & \multicolumn{2}{|c|}{ Hepatocytes } & \multirow{2}{*}{ Fluidity } \\
\hline & MSA & lysis & MSA & leakage & \\
\hline \multicolumn{6}{|l|}{ Hydrazines } \\
\hline Isonicotini acid & + & - & - & + & - \\
\hline Iproniazid & - & H & + & - & - \\
\hline Phenylhydrazine & + & + & H & - & + \\
\hline$p$-Tolylhydrazine & + & + & W & - & + \\
\hline \multicolumn{6}{|l|}{ Propinylamine } \\
\hline Deprenyl & $H$ & - & H & - & H \\
\hline Pargyline & + & - & + & - & - \\
\hline Clorgyline & H & Ht & + & H & H \\
\hline \multicolumn{6}{|l|}{ Others } \\
\hline Brofaromine & 世 & W & + & m & H \\
\hline Moclobemide & H & - & + & + & - \\
\hline
\end{tabular}

Effects of MAO inhibitors to biological membrane (erythrocytes and isolated hepatocytes) and DPPC liposomes. The grades of effects are: - , no effect; + , mild increase in membrane stabilizing action (MSA) and in hemolysis and leakage, and slight increase in membrane fluidity; H, moderate increase in MSA and in hemolysis and leakage, and mild increase in membrane fluidity; $\mathrm{H}$, prominent increase in MSA and severe increase in hemolysis and leakage and prominent increase in membrane fluidity.

moclobemide induced no apparent effect in the leakage of GPT from isolated hepatocytes, whereas treatment with $10^{-3} \mathrm{M}$ iproniazid or phenylhydrazine, or with $10^{-4} \mathrm{M}$ and $10^{-3} \mathrm{M}$ p-tolylhydrazine or pargyline significantly decreased GPT leakage below that of the control. Treatment with $10^{-5} \mathrm{M}$ clorgyline or brofaromine significantly decreased by GPT leakage, and treatment with $10^{-4}$ or $10^{-3} \mathrm{M}$ clorgyline or $10^{-3} \mathrm{M}$ brofaromine induced a remarkable increase in GPT leakage from isolated hepatocytes. These effects of MAO inhibitors on GPT leakage were similar to the effects on GOT leakage from the isolated hepatocytes.

After treatment with MAO inhibitors, isolated hepatocytes were dyed with trypan blue and the morphological changes were observed by phase contrast microscopy. Non-treated isolated hepatocytes were clearly round shaped, and few could be dyed with trypan blue, that is, most hepatocytes were viable (data not shown).

The decline of phase transition temperature of each MAO inhibitor was plotted against the control without MAO inhibitors (Fig. 2). The phase transition temperature of control liposomes was $41.74 \pm 0.05^{\circ} \mathrm{C}$ (mean $\pm \mathrm{SD}$ ). DPPC liposomal suspension in $10^{-3} \mathrm{M}$ iproniazid or moclobemide induced a slight decline in phase transition temperature. DPPC liposomal suspension in $10^{-3} \mathrm{M}$ p-tolylhydrazine depressed the phase transition temperature to $40.80^{\circ} \mathrm{C}$. Moreover, DPPC liposomes in $10^{-4}$ to $10^{-3} \mathrm{M}$ clorgyline, deprenyl or brofaromine remarkably depressed the phase transition temperature. The calculated concentrations of isonicotinic acid and iproniazid necessary to induce a $1{ }^{\circ} \mathrm{C}$ decline of the phase transition temperature were $1.29 \times 10^{-2} \mathrm{M}$ and $4.80 \times 10^{-2} \mathrm{M}$, respectively. The necessary concentration of brofaromine was only $1.73 \times 10^{-4} \mathrm{M}$, which was less than the $3.02 \times 10^{-4} \mathrm{M}$ of clorgyline.

All effects of MAO inhibitors on the erythrocytes, isolated hepatocytes, and DPPC liposomes are summarized in Table 3. Membrane stabilizing action (MSA) was evaluated from 
the decrease in hemolysis and enzyme leakage. Membrane fragility was evaluated from the increase in hemolysis and enzyme leakage from hepatocytes. Fluidity was evaluated from the decline of the phase transition temperature of the liposomal bilayer. We propose the degree of MSA, lysis, leakage and fluidity to be four grades. Hydrazine derivatives such as iproniazid and phenylhydrazine induced increase of hemolysis. However, these derivatives induced a decrease of the leakage of enzymes from isolated hepatocytes, which indicates membrane stabilizing action. Moclobemide and propinylamine derivatives such as deprenyl and pargyline stabilized the action of erythrocyte and isolated hepatocyte membranes. However, the effects of low concentrations of clorgyine and brofaromine were opposit to the effects of high concentrations. At low concentrations, they both induced a decrease in low hemolysis and enzyme leakage; at high concentrations they greatly enhanced these actions. Phase transition of DPPC liposomes that contained brofaromine occurred at the lowest temperature. Deprenyl and clorgyline also induced a decline of phase transition temperature to below the temperature of $p$-tolylhydrazine and phenylhydrazine. Other MAO inhibitors had no apparent effect on the phase transition temperature of DPPC liposomes.

\section{Discussion}

The basic structure of cell membranes, including erythrocytes and hepatocytes, is a phospholipid bilayer with a hydrophilic surface and a lipophilic portion inside ${ }^{13)}$. Severe membrane actions induced by some lipophilic medicines such as local anaesthetic agents and tricyclic antidepressants are reported to cause cytotoxicity ${ }^{\times, 11)}$. In the present study, membrane actions of MAO inhibitors against hypotonic hemolysis of erythrocytes and isolated hepatocytes indicated three patterns: stabilizing effects, leakage effects and combined effects.

In this study, hydrazine derivatives such as phenylhydrazine and p-tolylhydrazine at high concentrations gradually enhanced hemolysis. Phenylhydrazine is well known to cause hemolysis both in vitro and in vivo ${ }^{15,16)}$. The formation of hydrogen peroxide in erythrocytes has been demonstrated to be associated with the hemolytic action of phenylhydrazine ${ }^{1 i)}$. These results of the present study support the idea that hydrogen peroxide is formed at concentrations that exceed the detoxication capacity of erythrocytes, and this partly contributes to the cytotoxicity of phenylhydrazine ${ }^{16)}$. In the assay of biochemical activity in freshly isolated hepatocytes, phenylhydrazine and $p$-tolylhydrazine showed only a membrane stabilizing action. These phenomena were morphologically evident; that is, a few isolated hepatocytes of phenylhydrazine and $p$-tolylhydrazine treatments took up trypan blue but most hepatocytes were viable (data not shown). More recently, we reported that these inhibitors decrease the protein synthesis in primary cultured hepatocytes of rat $^{18)}$. Therefore, the decrease in GOT and GPT leakage in phenylhydrazine and p-tolylhydrazine treatments might be partly due to the suppression of synthesized proteins. Iproniazid also induced slight membrane damage in erythrocytes, whereas it slightly stabilized hepatocyte membranes. It seems that these various effects of hydrazide derivatives on biological membranes were caused by differences in the phospholipid constituents of the membranes of erythrocytes and isolated hepatocytes. From the determination of the phase transition temperature, these inhibitors had no effects on the fluidity of DPPC liposomes. Therefore, their membrane actions may have induced some influence on the proteins of the cell membranes but not on the phospholipids.

Brofaromine and clorgyline exerted the maximum membrane stabilization at the lowest concentrations. Depression of the phase transition temperature of DPPC liposomes by bro- 
faromine and clorgyline indicates that these agents partly penetrate the bilayers and increase the fluidity of the bilayer membrane. In model membranes of DPPC liposomes, they are crystalline below the phase transition temperature $\left(41.0^{\circ} \mathrm{C}\right)$ and in a liquid crystalline phase above that temperature. In general, the acyl chains of phospholipids are arranged in parallel with slight mobility in the crystalline phase. In the liquid crystalline phase, the disorder in the conformation of the lipid tails is characterized by increased gauche rotations, that is, their acyl chains increase mobility and thus the lipid bilayer becomes thin ${ }^{19)}$. In addition, membrane fluidity is influenced not only by temperature but also by various environmental factors, such as chemical compounds or $\mathrm{pH}^{20,21)}$. An increase in fluidity of the bimolecular membrane of phospholipid induces expansion of the bilayer membrane ${ }^{22,23)}$. Therefore, we can imagine that the volume can increase because the cell membrane of erythrocytes and hepatocytes is expanded by treatment with brofaromine and clorgyline, and so more water remains in these cells until lysis and leakage occur. In this study, the two MAO inhibitors, at concentrations below those causing lysis and leakage, substantially decreased hemolysis and enzyme leakage. From these results, the increase in membrane fluidity by brofaromine and clorgyline is one reason for their stabilizing effects on the erythrocyte and hepatocyte membranes. However, it is important to realize that if the fluidity of cell membranes is increased too much, cell membranes overexpand and become unstable. Thus, brofaromine and clorgyline exerted lytic and leakage effects on the erythrocytes and hepatocytes at higher concentrations. The effects of clorgyline were more severe than those of brofaromine. However, the phase transition temperature of DPPC liposome in clorgyline was higher than that in brofaromine at $10^{-3} \mathrm{M}$. That is, the increase in the fluidity of DPPC liposomes by clorgyline was less than that by brofaromine. Thus, these membrane actions may affect not only phospholipids, but also other components of the cell membranes, such as protein. Morphological features of isolated hepatocytes treated with these MAO inhibitors induced bumpy surfaces within trypan blue; and at $10^{-3} \mathrm{M}$, decreased viable cell numbers and the amorphous remains of hepatocytes were observed (data not shown). The fact that the low concentration of brofaromine and clorgyline was enough to depress the phase transition temperature indicates their marked stabilizing effect on the cell membranes compared to other MAO inhibitors. This is similar to the action of potent local anesthetics such as tetracaine or bupivacaine, which induce a marked decline of the phase transition temperature and increase in the stabilization of the cell membrane, as opposed to weak local anesthetics such as procaine ${ }^{24)}$. After single oral administrations of brofaromine $(100 \mathrm{mg})$ to healthy volunteers, the maximum plasma concentration of brofaromine reaches about $2 \times 10^{-6} \mathrm{M}^{25)}$. In this study, treatment of erythrocytes and isolated hepatocytes with $10^{-6}$ to $10^{-4} \mathrm{M}$ brofaromine stabilized the cell membranes. This suggests that exposure to brofaromine at a plasma concentration of $10^{-6} \mathrm{M}$ does not induce a clinical effect on the cell membrane.

In conclusion, the present study showed that MAO inhibitors possess an ability to produce various membrane effects. The potency of MAO inhibitors on hypotonic hemolysis and freshly isolated hepatocytes was clorgyline $>$ brofaromine $>$ other MAO inhibitors. These results suggest that brofaromine induces membrane damage at high concentrations, thus it should be used carefully in clinical applications.

\section{References}

1) Popper $\mathrm{H}$ : Pathological findings in jaundice associated with iproniazid and related compounds. Ann NY Acad Sci, 80: 873-884 (1959) 
2) Bandt $\mathrm{C}$ and Hoffbauer FW: Liver injury associated with tranylcypromine therapy. JAMA, 25: 752-753 (1964)

3) Rees L: Drugs used in the treatment of psychiatric disorders. Abstr World Med, 39: 129 (1966)

4) Ebert MH and Shader RI: Hepatic effects. In: Psychotropic Drug Side Effects; Clinical and theoretical considerations, Shader RI and DiMascio A (Eds), Williams and Wilkins, Baltimore, pp 175197 (1970)

5) Blackweil B: Adverse effects of antidepressant drugs. Part 1: Monoamine oxidase inhibitors and tricyclics. Drugs, 21: 201-219 (1981)

6) Zimmerman HJ: Psychotropic and anticonvulsant agents. In: Hepatotoxicity. Zimmerman HJ (Ed), Appleton-Century-Crofts, New York, pp 395-417 (1978)

7) Johnson JP: Some observations upon a new inhibitor of monoamine oxidase in brain tissue. Biochem Pharmacol, 17: 1285-1297 (1968)

8) Yasuhara H, Tonooka M, Kamei K and Sakamoto K: Membrane effects of various drugs on isolated rat hepatocyte and erythrocytes. Toxicol Appl Pharmacol, 79: 453-460 (1985)

9) Arakawa K, Tonooka M, Goto $\mathrm{H}$ and Sakamoto K: Membrane stabilizing action of NCO-650 and its congeners. Jpn J Pharmacol, 36: 311-318 (1984)

10) Tsuji M, Kodama K and Oguchi K: Protective effects of S-adenosyl-L-methionine against enzyme leakage from cultured hepatocytes and hypotonic hemolysis. Jpn J Pharmacol, 52: 45-49 (1990)

11) Segien PO: Preparation of isolated rat liver cells. Methods Cell Biol, 13: 29-83 (1976)

12) Ohkawa J, Miyoshi Y, Matsuura $S$ and Misaki $H$ : The new colorimetric assay for the determination of transaminase activity with pyruvate oxidase. Jpil J Clin Pathol, Suppl. 26: 70 (1978) (in Japanese)

13) Singer SJ and Nicolson GL: The fluid mosaic model of the structure of cell membranes. Science, 175: $720-731$ (1972)

14) Yasuhara $H$, Matsuo $H$, Sakamoto $K$ and Ueda I: Mechanism of membrane stabilizing and lytic effects of tricyclic antidepressants. Jpn J Pharmacol, 30: 397-401 (1980)

15) Jandl JH, Engle LK and Allen DW: Oxidative hemolysis and precipitation of hemoglobin. I. Heinz body anemias as an acceleration of red cell aging. J Clin Invest, 39: 1818-1836 (1960)

16) Jain SK and Hochstein P: Generation of superoxide radicals by hydrazine its role in phenylhydrazine-induced hemolytic anemia. Biochim Biophys Acta, 586: 128-136 (1979)

17) Cohen $\mathrm{G}$ and Hochstein P: In vivo generation of $\mathrm{H}_{2} \mathrm{O}_{2}$ in mouse erythrocytes by hemolytic agents. J Pharmacol Exp Ther, 147: 139-143 (1965)

18) Tsuji M, Kodama K, Okazaki M, Yasuhara H and Oguchi K: Hepatic toxicity of monoamine oxidase inhibitors in isolated and primary cultured hepatocytes of rat. In: Monoamine Oxidase: Basic and Clinical Aspects. Yasuhara H, Parvez SH, Sandler M, Oguchi K and Nagatsu T (Eds), VSP Press, Holland (1992) (in press)

19) Gulik-Krzywicki T: Structural studies of the associations between biological membrane components. Biochim Biophys Acta, 415: 1-28 (1975)

20) Shimshick EJ and McConnel HM: Lateral phase separation in phospholipid membranes. Biochemistry, 12: 2351-2360 (1973)

21) Wu SHW and McConnel HM: Phase separations in phospholipid membranes. Biochemistry, 14: $847-854$ (1975)

22) Isomaa B: Interactions of surface-active alkyltrimethyl-ammonium salts with the erythrocyte membrane. Biochem Pharmacol, 28: 975-980 (1979)

23) Ueda I: Interfacial effects of anesthetics on membrane fluidity. In: Advances in Membrane Fluidity. Roland CA, Cyril CC and Larry MG (Eds), Wiley-Liss, New York, Vol 5, pp 15-33 (1991)

24) Ueda I, Tashiro $C$ and Arakawa K: Depression of phase transition temperature in a model cell membrane by local anesthetics. Anesthesiology, 46: 327-332 (1977)

25) Hengen N, Jedrychowski $M$ and Hoffmann E: Pharmacokinetics of CGP11305A in man after acute and prolonged oral treatment. In: Monoamine Oxidase and Disease. Tipton KF, Dostert $\mathrm{P}$ and Benedetti MS (Eds), Academic Press, London, pp 185-191 (1984)

[Received June 23, 1992: Accepted July 15, 1992] 